\title{
Temperature Measurement and Numerical Simulation of Semiconductor Bridge in Constant Current
}

\author{
Wenchao Zhang ${ }^{1 \text { a* }}$, Xiaowei Wang ${ }^{1 \text { b }}$, Guihua Wang ${ }^{2 \mathrm{c}}$, Baoqing Yin ${ }^{1 \mathrm{~d}}$ \\ ${ }^{1}$ School of Chemical Engineering, Nanjing University of Science and Technology, Nanjing 210094, \\ China \\ ${ }^{2}$ School of Electronic and Optical Engineering, Nanjing University of Science and Technology, \\ Nanjing 210094, China \\ ae-mail :zhangwenchao303@yahoo.com.cn, ${ }^{b}$ e-mail :wangxiaowei19877@163.com \\ ce-mail :ghwang@mail.njust.edu.cn, de-mail :gmyinbaoqing@gmail.com
}

\begin{abstract}
Keywords: Semiconductor Bridge; Steady-state Model; Infrared Thermal Imaging; Safety; Electroexplosive Devices
\end{abstract}

\begin{abstract}
The temperature of electric initiating device under constant current has the vital significance to security and firing performance of electrical explosive initiator. Semiconductor bridge which is representative in electrical explosive devices has been widely used for excellent performances. In this study, the heavily doped polysilicon thin film was chosen as semiconductor bridge. Temperature changes of semiconductor bridge under different currents were measured using the infrared microscopic thermographer. The experimental results show that the maximum temperature of semiconductor bridge ascends while current rises from $0.70 \mathrm{~A}$ to $0.90 \mathrm{~A}$. Based on the data analysis of infrared temperature measurement, steady-state mathematical model $\rho c \frac{d T}{d t}=I U-\lambda_{\text {con }}\left(T-T_{0}\right)-A \varepsilon \sigma\left(T^{4}-T_{0}^{4}\right)$ of semiconductor bridge was established. Through consumed time and temperature at thermodynamic equilibrium, the thermal conductivity and heat capacity of semiconductor bridge were determined and the simplified formula $T_{n}=\frac{I^{2} R(T) \Delta t+\rho c T_{n-1}+\lambda_{\text {tot }} T_{0} \Delta t}{\rho c+\lambda_{\text {tot }} \Delta t}$ was obtained. Then the simulation curve of temperature changes at different current is generated by corresponding calculation procedure. The simulated curve is in good agreement with experimental results.
\end{abstract}

\section{Introduction}

Semiconductor bridge (SCB) as a transducer of electronic energy to thermal energy which was originally conceived by Hollander[1] in 1968 and further developed by Benson[2] et al. is an advanced electro-explosive devices (EED's) fabricated using integrated circuit technique[3].The SCB of electro-explosive devices has a wide of applications in the areas such as automotive airbags, rocket ignition, and various ordnance system[4] to provide the igniting of energetic compounds for its improved firing performances in terms of high safety, low ignition energy and short ignition time[5,6]. Moreover, the fabrication process for SCB is fully compatible with standard microelectronics processing technology, opening the door to integrate the ignitors into the logic circuits or Micro Electronic Mechanical System (MEMS) and enhancing the functional ignitors for MEMS[7,8]. Therefore, the SCB has attracted attention from many researchers for its improved performance mentioned above.

So far many researchers have done a lot of work mainly focusing on the investigation of firing performance of SCB. Benson et al.[2] found the phenomenon of vaporization process and plasma formation for SCB under the current plus applied to metal electrode. Kim et al.[7-9]employed the microwave resonator probe to investigate the the behaviors of plasma generated by SCB such as the plasma density, the propagation speed for plasma and the plasma size with aid of the visualization of micro scale plasma and obtained the temperature of plasma through the optical emission 
spectra(180-700 nm)[10]. Furthermore, Park et al found that the peak of the single-SCB preceded that of the poly-SCB during the plasma generation process, indicating that the rising rate of the temperature with the single-SCB was higher than that of poly-SCB[8].

However, there is little data on bridge area temperature as a function of constant current acting on SCB. The safety of the SCB igniting device determines the safety of the whole ignition chain. Especially the temperature variation laws of bridge area with constant current acting on SCB can supply important information for estimating the firing current and safety current of SCB. There are many methods used to measure temperature. Compared with other methods, the infrared thermograpy exhibits in terms of advantages such as non-destructive, high accuracy, no interference and low energy loss from the object[11-13]. So the purpose of this paper is aid to report the temperature measurement of bridge area for SCB under the constant current with the infrared microscopic thermographer and put forward the steady-state mathematical model to simulate temperature variation of micro-bridge area based on the experimental data analysis, providing the quantitative evidence of SCB's safety.

\section{Experiment}

The structure of SCB used in this study was shown in Fig.1. The fabrication of SCB was realized with standard microelectronics processing technology as follows: Firstly, the polysilicon membrane doped phosphor with concentration of $10^{20} / \mathrm{cm}^{3}$ for SCB was obtained by the epitaxial growth of silicon oxide through high temperature oxidation of silicon substrate. Then the process of metal sputtering was employed to attach the two metal lands of $\mathrm{Au}$ on the polysilicon membrane, realizing the function of electrode with the current passing through the SCB devices. The average value of resistance for SCB was $1 \Omega$ and the dimensions for bridge area of SCB is $300 \mu \mathrm{m} \times 90$ $\mu \mathrm{m} \times 2 \mu \mathrm{m}(\mathrm{W} \times \mathrm{L} \times \mathrm{T})$.



Fig.1 Schematic view of a typical SCB

The experiments of measuring the temperature variation and heat generating process for bridge area of SCB under the constant current were carried out with infrared radiation thermometers. The instrument used was Thermo Vision A40-M infrared radiation thermometer (FLIR SYSTEM COMPANY) with an $18 \mu \mathrm{m}$ microlens.

Temperature rising process of sample was recorded by infrared radiation thermometers and stored in the computer, then the process recorded was analyzed via the ThermaCAM Researcher software accompanied with infrared radiation thermometer and the data of temperature dependence on time was obtained. The process of measuring the temperature was as follows: firstly, SCB was aimed at infrared microscopic thermometer, secondly, the current and conduction time of constant current source were set, then the software installed in the computer was started while current acting on SCB for recording the infrared image of conduction process.

The principle based on infrared thermal image is the Stefan- Boltzmann law of thermal radiation, the key factor on accuracy of measuring temperature is the emissivity of materials. A simple method determining the emissivity of bridge area was employed as follows: The measured object(a) and another object(b, whose emissivity was known) were heated together up to $70{ }^{\circ} \mathrm{C}$ higher than room temperature. The thermal images of $a$ and $b$ were recorded as shown in Fig.2. The emissivity 
value was the real emissivity of a at the moment when the temperature of a was consistent with that for $b$ through adjusting the emissivity of a. With this method, the emissivity for SCB used in the experiment is 0.41 .

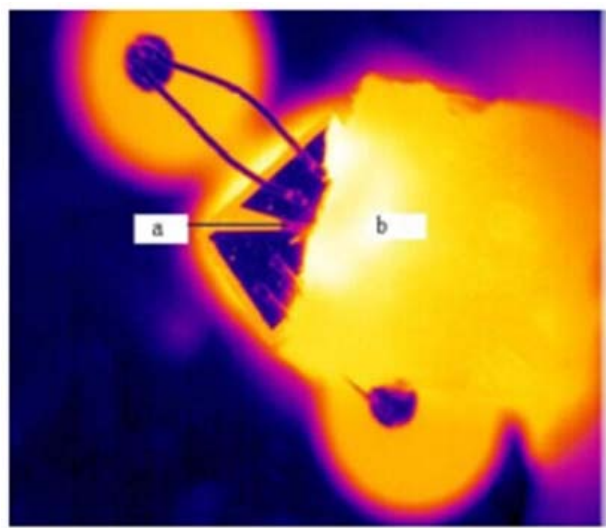

Fig.2 FLIR images measuring temperature of $a$ and $b$ together under heating

Fig. 3 shows the FLIR temperature distribution for SCB at some time. It could be observed that the variation process of temperature for bridge area reflects the heat generating process for bridge area as the core component of SCB. So the experiments of FLIR measuring the temperature variation of bridge area under different constant currents were carried out for obtaining the evidence of supporting safety basis of SCB and the variation curves of temperature with conduction time under different currents (0.7 A, 0.8 A, 0.9 A respectively) were obtained as shown in Fig.4. It could be found that the temperature of bridge area rises with increasing of constant current and the temperature is up to $332{ }^{\circ} \mathrm{C}$ for current being 0.9 A. Besides, the phenomenon in Fig. 4 that the temperature of SCB keeps constant from $250 \mathrm{~s}$ to $300 \mathrm{~s}$ indicates that the thermodynamical equilibrium is realized and the time of realizing thermodynamical equilibrium becomes longer from the slope of the curves. In order to get deep understanding of temperature behaviors for bridge area with SCB, a model was put forward to give the interpretation of temperature variation laws for bridge area supporting the predication of safety basis for SCB.

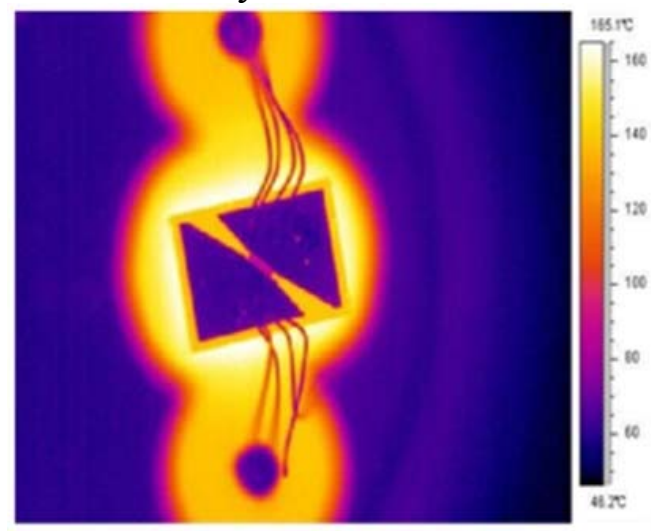

Fig.3 FLIR images measuring temperature distribution of SCB under constant current of $0.7 \mathrm{~A}$

\section{Steady-State Model for Temperature Behavior of SCB}

The analysis of the simplified steady-state model describing the temperature behavior of SCB was presented. The joule heating effect on SCB caused by constant current passing through the polysilicon membrane led to the temperature rise for bridge area of $\mathrm{SCB}$, while the heat diffusing via the substrate and metal electrode in the means of heat conduction and thermal radiation occurred during the temperature rising of SCB. Meanwhile, a large number of parameters with SCB such as heat capacity, thermal conductivity and thermal emissivity were involved and only obtained by experiment. So the temperature variation was a complex physical process influenced by many factors. In order to get temperature variation information conveniently, some hypotheses about the steady-state model should be made as follows:

(1) The system in the steady-state model included the silicon substrate and polysilicon 
membrane of SCB, in which the plug with large volume possessed more radiating area than that of the chip with $\mathrm{SCB}$ ( the polysilicon membrane mentioned above ). And the boundary conditions for partial differential equation describing the steady-state model were obtained by the relationship between SCB chip and plug.

(2) The factor of the joule heating effect on wire and silicon substrate was ignored because the thickness of SCB was smaller compared to that of silicon substrate and current only acted on the bridge region of SCB.

(3) Because the plug contacted the bridge region closely, ignoring the contact thermal resistance and heat capacity of interface between bridge region and plug. The interface of the plug with bridge region for SCB exhibited the same temperature. Therefore, the heat-transfer between bridge area of SCB and the plug via the radiation was neglected as well.

(4) The physical parameters with the plug and SCB such as thermal conductivity, density, specific heat capacity and the emissivity kept constant during the whole temperature rising process. Moreover, the effect of thermal radiation should be considered during the heat exchange process.

(5) During the heat transfer process, the energy exchange existed between the air and heating object and the energy was in proportion to the temperature. So the part of heat exchange could be considered in the heat conduction without calculating it individually.

During the process of heat generating on SCB when the biased voltage was applied to chip through the function of metal electrode, the energy exchange occurred in means of heat transfer, thermal radiation. So the equation based on the energy conservation and the assumption mentioned above was put forward as follows:

$$
\rho c \frac{d T}{d t}=I U-\lambda_{\text {con }}\left(T-T_{0}\right)-A \varepsilon \sigma\left(T^{4}-T_{0}^{4}\right) .
$$

Where $\rho$ is the density of bridge region, $\mathrm{kg} / \mathrm{m}^{3}$; $c$ is the heat capacity of SCB chip, $\mathrm{J} / \mathrm{K}$;

$I$ is the current applied to $\mathrm{SCB}, \mathrm{A}$; $U$ is the biased voltage acting on $\mathrm{SCB}, \mathrm{V}$;

$\lambda_{c o n}$ is the thermal conductivity coefficient of SCB chip, $\mathrm{W} / \mathrm{m} \cdot \mathrm{K}$;

$A$ is the surface area of chip, $\mathrm{m}^{2} ; \varepsilon$ is the emissivity of SCB region;

$\sigma$ is the Stefan-Boltzmann constant, $5.67 \times 10^{-8} \mathrm{~W} / \mathrm{m}^{2} \cdot \mathrm{K}^{4}$;

$T$ is the temperature of bridge region, $\mathrm{K} ; T_{0}$ is the initial temperature of bridge region, $\mathrm{K}$.

The key to solve this differential equation was the $c$ and $\lambda_{\text {con }}$ of SCB. However, there was little data available on the two parameters in the literature. Meanwhile, the heat exchange was a dynamic process. So it was necessary to determine these parameters via simplified calculation and experiment data. The $\lambda_{\text {con }}$ could be obtained by the condition that the value of equation (1)was equal to zero when the thermal equilibrium for SCB was realized. The heat exchange mainly existed between the chip and the substrate ignoring the thermal radiation at the beginning of small current applied to chip. So the equation (1) could be expressed as:

$$
\rho c \frac{d T}{d t}=I U-\lambda_{\text {con }}\left(T-T_{0}\right) \text {. }
$$

If $t$ is less than zero, the value of IU is zero. When $t$ is greater than zero, the solution of equation (2) could be written as follows:

$$
T=T_{0}+\frac{I U}{\lambda_{\text {con }}}\left(1-e^{-t / \tau}\right) \text {. }
$$

Where $\tau$ is the thermal time constant corresponding to the temperature response.

When the the thermal equilibrium of SCB was realized( $t$ is much larger than $\tau$ ), the equation (3) could be converted as:

$$
T=T_{0}+\frac{I U}{\lambda_{\text {con }}} \text {. }
$$

From the equation (3) and (4) the $\tau$ was obtained. Meanwhile, the relationship between $c$ and thermal conductivity coefficient $\lambda_{\text {con }}$ could be expressed the following formula: 


$$
\tau=\frac{c}{\lambda_{\text {con }}}
$$

Therefore, the value of $c$ was determined by the formula (5) utilizing the parameter $\lambda_{\text {con }}$ and $\tau$. The radiation thermal conductivity $\left(\lambda_{\text {rad }}\right)$ of SCB could be obtained for radiation energy increasing with the temperature rising through equation (1):

$$
\lambda_{\text {rad }}=\frac{d\left(A \varepsilon \sigma T^{4}\right)}{d T}=4 A \varepsilon \sigma T^{3} .
$$

As a result, the total thermal conductivity $\left(\lambda_{\text {tot }}\right)$ of SCB included $\lambda_{\text {rad }}$ and $\lambda_{\text {con }}$.

So the equation (1) could be transformed into a neat form:

$$
\rho c \frac{d T}{d t}=I^{2} R(T)-\lambda_{\text {tot }}\left(T-T_{0}\right) \text {. }
$$

For the small time interval the formula (7) could be further converted to the following form:

$$
\left(\rho c+\lambda_{\text {tot }} \Delta t\right) T_{n}=I^{2} R(T) \Delta t+\rho c T_{n-1}+\lambda_{\text {tot }} T_{0} \Delta t .
$$

Therefore, the expression related to the temperature of SCB under the constant current was obtained as follows:

$$
T_{n}=\frac{I^{2} R(T) \Delta t+\rho c T_{n-1}+\lambda_{t o t} T_{0} \Delta t}{\left(\rho c+\lambda_{\text {tot }} \Delta t\right)} .
$$

In order to get the simulation curves, the time interval of the experiment was used to calculate the temperature from the SCB and the $\lambda_{\text {rad }}$ was obtained by setting the parameter $T$ as $T_{0}$ with the equation (6). Moreover, $T_{n}$ corresponding to the temperature for different time was gotten by Eq.(8) for $\lambda_{\text {tot }}$ including $\lambda_{\text {rad }}$ and $\lambda_{\text {con }}$, then a computer program with digital processing of Eq.(6) and (8) was made to simulate the temperature variation of SCB without the complicated calculation mentioned above. So the simulation curves for temperature variation of SCB were obtained as shown in Fig.4 (0.7 A, 0.8 A, 0.9 A). From the Fig.4 it could be found that the simulation curves could reflect the temperature variation with time very well, and the maximum temperature errors between simulation curves and experiment curves is less than $10 \mathrm{~K}$. Especially when the temperature of SCB at latter time reached the maximum point indicating the thermal balance for chip of SCB was realized, the simulation curves was highly consistent with experiment curves. Therefore, the steady-state model can be used to predict the temperature variation very well, providing the important information about the temperature behavior for SCB and evaluating the safety for SCB.

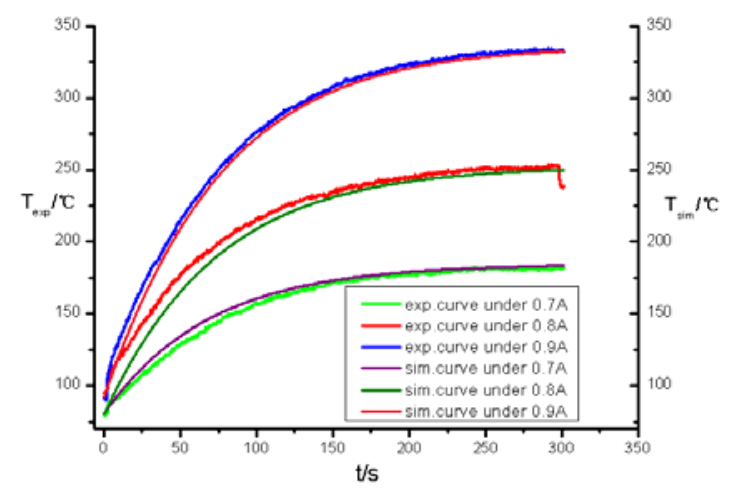

Fig.4 Time evolutions of the measured and simulated temperature

\section{Conclusion}

This paper reports the temperature variation behavior of SCB under the different constant currents with the infrared radiation thermometers and the steady-state model proposed based on the 
experiment data simulating the temperature variation. It could be observed that the data of numerical simulation exhibited great agreement with the experimental curves, indicating that the steady-state model could be used to predict the temperature variation very well. Therefore, the steady-state model based on the experiment data analysis could be applied to make the prediction of temperature variation laws, providing the quantitative evidence of evaluating the safety for SCB

and guidelines for investigating the temperature behaviors and heat generation of SCB under the other forms of input energy such as plus current and radio frequency.

\section{Acknowledgment}

This work was supported by NSFC under Grant No. 50806033.

\section{References}

[1] J.R. Hollander and E. Lewis, U.S. Patent 3,366,055, (1968)

[2] D.A. Benson, M.E. Larsen, A.M. Renlund, W.M. Trott and R.W. Bickes: J. Appl. Phys Vol. 62 (1987), p. 1622

[3] T. Vijayan and V.K. Rohatgi: IEEE. Trans. Plasma. Sci Vol. 13 (1985), p. 197

[4] J. Kim, K.S. Nam and K. Jungling: IEEE. Trans. Electron. Devices Vol. 44 (1997), p. 1022

[5] S. Harris and S. Porter: Safe. Assoc (1998)

[6] J. Kim, E. Schamiloglu, B. Martinez-Tovar and K.C. Jungling: Electron. Lett Vol. 30 (1994), p. 603

[7] J.U. Kim, C.O. Park, M.I. Park, S.H. Kim and J.B. Lee: Phys. Lett. A Vol. 305 (2002), p. 413

[8] M.I. Park, H.T. Choo, S.H. Yoon and C.O. Park: Sens. Actuators. A Vol. 115 (2004), p. 104

[9] J. Kim, E. Schamiloglu, B. Martinez-Tovar and K. Jungling: IEEE. Trans. Instrum. Meas Vol. 44 (1995), p. 843

[10] J. Kim, T.M. Roh, K.I. Cho and K.C. Jungling: IEEE. Trans. Electron. Devices Vol. 48 (2001), p. 852

[11] S. Datcu, L. Ibos, Y. Candau and S. Matte: Infrared. Phys. Technol Vol. 46 (2005), p. 451

[12] C.J. Ming and Y. J. Hsien: J. Quant. Spectrosc. Radiat.Transfer Vol. 56 (1996), p. 133

[13] G. Kastberger and R. Stachl: Behav. Res. Methods. Instrum. Comput Vol. 35 (2003), p. 429 